\title{
Wavelet-Based Classification of Remotely Sensed Images: A Comparative Study of Different Feature Sets in an Urban Environment
}

\author{
J. Chen ${ }^{1}$, D. Chen ${ }^{2 *}$, and D. Blostein ${ }^{1}$ \\ ${ }^{1}$ School of Computing, Queen's University, Kingston, Ontario, Canada \\ ${ }^{2}$ Department of Geography, Queen's University, Kingston, Ontario, Canada
}

\begin{abstract}
This paper presents a series of experiments on classification of remotely sensed images, to investigate the effectiveness of various combinations of different types of feature sets, including spectral features, variance features and wavelet-based features. All the experiments use the identical study area, training data, reference data, testing data, and classification algorithm while varying the feature sets. The classification accuracy from different feature sets is evaluated using the traditional accuracy assessment from reference data. The experimental results show that the spectral-based feature set has the basic discrimination power to distinguish classes with middle and high homogeneity value. However, it has little success in correctly classifying classes with low homogeneity value, such as the residential class. Compared with spectral features, the multi-scale wavelet-based feature set can improve the discrimination power for classes with both low and high homogeneity value. The variance-based feature set alone has little discrimination power, no matter what homogeneity level the class has. However, adding the variance features into the spectral feature or wavelet-based feature set can dramatically increase the classification accuracy for classes with low homogeneity value.
\end{abstract}

Keywords: Remote sensing, image classification, wavelet, land use, land cover, feature extraction

\section{Introduction}

Classification of a remotely-sensed image is the process of assigning categorical classes (such as land-use/land-cover types) to the pixels in the digital remotely-sensed images (Lillesand, 2000). Automatic classification of remotely-sensed images plays an increasingly important role in analyzing the huge number of remotely-sensed images. Various classification approaches have been applied to the classification of remotely-sensed images in the past decades. The approaches are characterized as supervised and unsupervised classifications (Jensen, 1996). Supervised classification uses a set of predefined classes, and labeled training data. In contrast, unsupervised classification works with little prior knowledge and aims to discover natural clusters that exist in the data set. We focus on supervised classification in this paper.

The most commonly used supervised classification approach is the pixel-based spectral approach, in which a classifier is applied to label a pixel based on its spectral values in different bands. The pixel-based classification approach has been criticized for not considering the spatial structure (or heterogeneity) of a class (Chen et al., 2003), especially for complicated classes in an urban environment when fine spatial resolution imagery is used (Gong and Howarth, 1990; Fung and Chan, 1994). For example, a single-family house may be represented by a pixel in a 30-m spatial resolution remotely sensed image. However, when looked at in 4-m spatial resolu-

\footnotetext{
* Corresponding author: chendm@queensu.ca
}

tion images, a single-family house may include building roof, pool, shrubs, grassland and driveway, etc. Consequently, a single-family residential house cannot be accurately classified by using the spectral signatures of individual pixels.

Considerable research has been conducted to increase the accuracy of the spectral analysis of remotely-sensed images by using texture analysis, in which some measure of variability in density values is estimated within local windows. The most common of these are contrasts between neighboring pixels (Edwards et al., 1988), the standard deviation (Arai, 1993), or local variance (Woodcock and Harward, 1992). Other measures include the coefficient of variation, higher moments of different order, spatial auto-correlation, and greylevel differences. Conners and Harlow (1980) proposed the gray level difference histograms in which various texture features are calculated to classify texture. Second-order statistical based methods rely on the spatial arrangement of adjacent pixels that consider the second order probability distribution. However, most of the studies primarily focus on the use of texture on a single scale, while the textures of a class in the real world are often non-uniform and can be characterized at multiple scales (Pathak and Dikshit, 2007).

Wavelet-based classification addresses of the lack of adequate tools to represent textures at different scales (MyInt, 2000). Several researchers showed that texture analysis of the wavelet decomposed sub-images at different scales provides superior discrimination of different urban objects/samples (Zhu and Yang, 1998; MyInt, 2002). Zhu and Yang (1998) achieved high accuracies in distinguishing different texture 


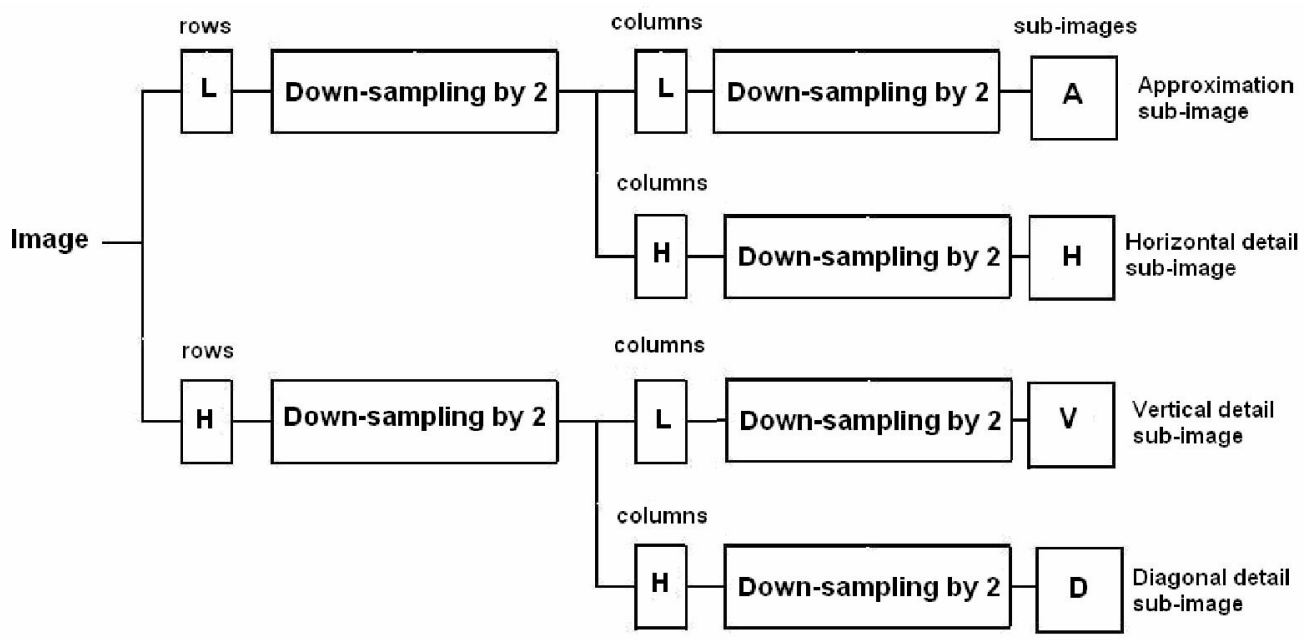

(a)

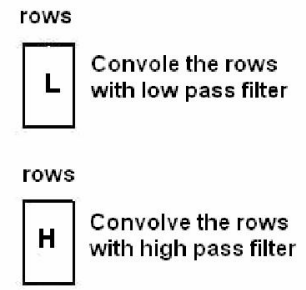

(b)
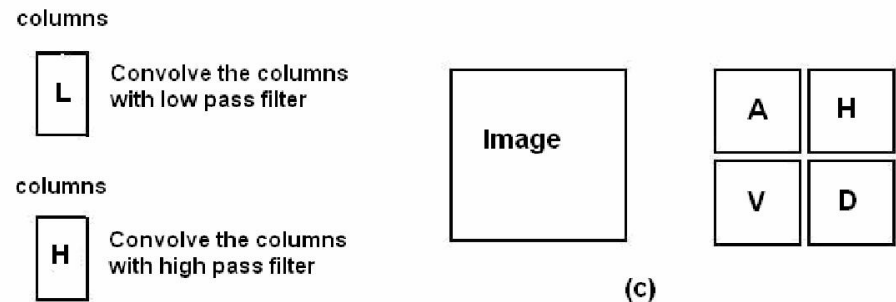

(c)

Figure 1. (a) Image convolution process; (b) Low-pass filter and high-pass filter convolution for rows and columns; (c) Input Image and the output sub-images (A, H, V, D) and their positions in the output image.

images. Chang and Kuo (1993) proposed a multi-resolution approach based on a modified wavelet transform called the tree-structured wavelet transform for the texture analysis and the classification. Andrew and Jian (1993) studied the texture classification using wavelets. They evaluated wavelets for the classification of twenty-five natural textures selected from the Brodatz album (1966), which is an album consisting of various types of texture images. Unser (1995) proposed a new approach for texture classification and segmentation using the wavelet frames. Yet individual assessment of the effectiveness of wavelet-based approaches using different wavelet features measures without having integrated assessment of wavelets with other non-wavelet-based feature sets has sparked a marked interest on advanced study.

The objective of this paper is thus to investigate the effectiveness of three feature sets (spectral-based feature set, variance-based feature set, wavelet-based feature set) and the effectiveness of combining two other feature sets with wavelet-based features. To achieve this goal, we carry out a series of classification experiments using combinations of three different feature sets, while keeping the training data, reference data, and classification algorithm unchanged. In the following, we describe the wavelet-based feature decomposition first. Then the methodology is presented.

\section{Wavelet-Based Classification}

Wavelet-based classification is developed based on the wavelet theory initially developed from the signal processing using a set of special signals, which are oscillatory (look like waves) and have amplitudes decaying to zero in both positive and negative directions (Young, 1993). In the discrete wavelet transform (DWT), the signal is passed through a series of lowpass filters to analyze the low frequencies, and a series of high-pass filters to analyze the high frequencies. The resolution of the signal, which is a measure of the amount of detailed information in the signal, is changed by the filtering operation, and the scale is changed by the up-sampling or down-sampling operations. After passing the signal through a half band low-pass filter, half of the samples can be eliminated. Therefore, the resolution is halved after the filtering operation. The DWT analyzes the signal at different frequency bands with different resolutions by decomposing the signal into a coarse approximation and detail information.

The discrete wavelet transform proposed by Mallat (1989) initially decomposes an image into one approximation image and three detail images. It filters the original image with complementary low-pass and high-pass filters in each dimension. The filtered images are down-sampled at every other pixel, 


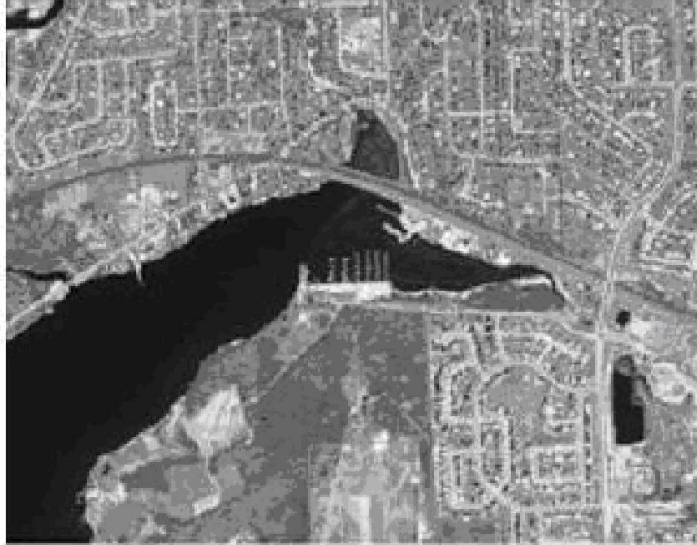

(a) Original image

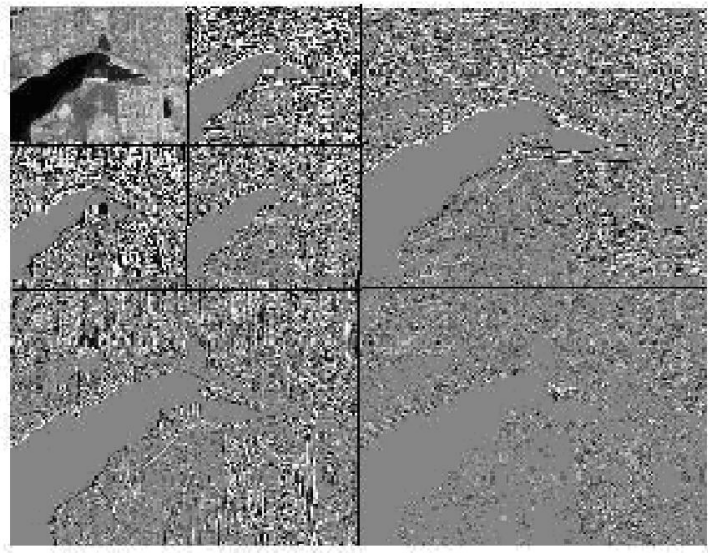

(c) Two level wavelet decomposition

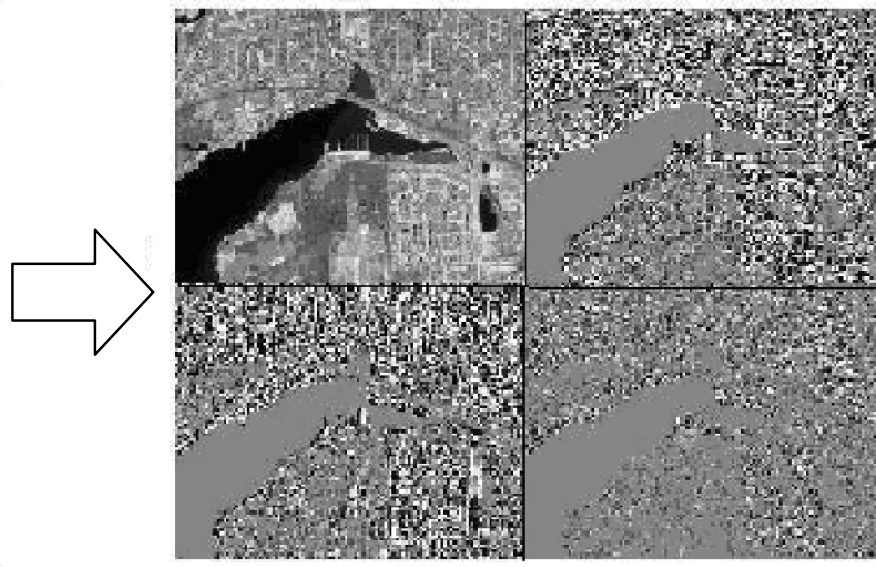

(b) One level wavelet decomposition

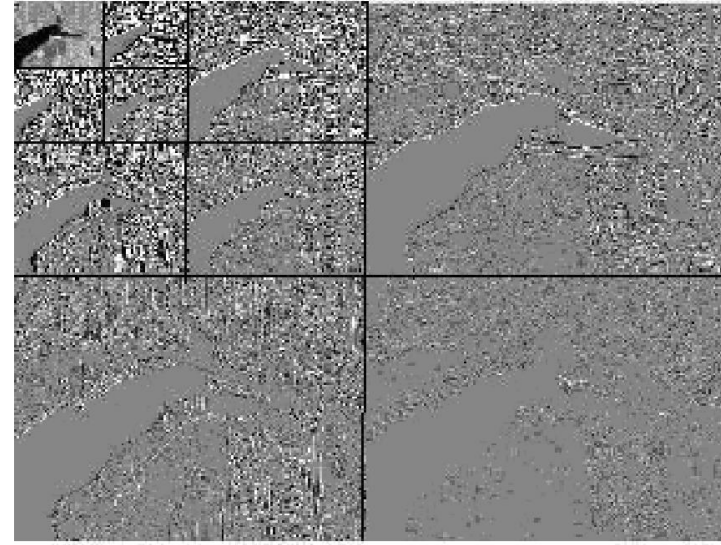

(d) Three level wavelet decomposition

Figure 2. An example of one-, two-, and three-level wavelet decompositions using Haar wavelet.

producing four images of half the resolution of the original. For a one-level wavelet transform, the result is an approximation sub-image and three detail sub-images. The approximation sub-image maintains the major trend of the original image, while the detail sub-images represent the difference between the approximation sub-image and the original image in different directions (Bian, 2003).

The process of wavelet decomposition can also be described as a filter convolution with the original image. For the level one wavelet decomposition, the signals can be obtained by convolving the signal with the appropriate filters. Figure 1 illustrates the process of filter convolution with the image.

The filter process can be applied again to the approximation sub-image (or any detail sub-images), resulting in four additional second-level sub-images (See Figure 2). There are many different types of wavelets, for example, Haar wavelet, Daubechies, coilets, symlets, biorthogonal (Gonzalez and Woods, 2001). The Haar wavelet is the simplest and oldest one in the wavelet family. For the Haar wavelet, the low-pass filter coefficient in a sub-image is obtained by dividing the sum of two adjacent pixel values by $\sqrt{2}$, whereas the highpass filter coefficient is obtained by dividing the difference of two adjacent pixels value by $\sqrt{2}$. The approximation subimage from a simple Haar transform at the second-level is the average of the approximation at the first-level, while the detailed sub-images represent differences between approximations at two successive levels. This filtering process creates a multi-level representation of the original image through two sets of information. One is a hierarchy of approximation at increasingly coarser resolutions and the other is detail at corresponding resolutions. Thus, the wavelet transform not only separates the trend from details as many other filtering methods do, but it also presents both sets of approximation and detail sub-images. Figure 2 illustrates the standard wavelet decomposition process of an image at one, two and three levels using a simple Haar transform. 


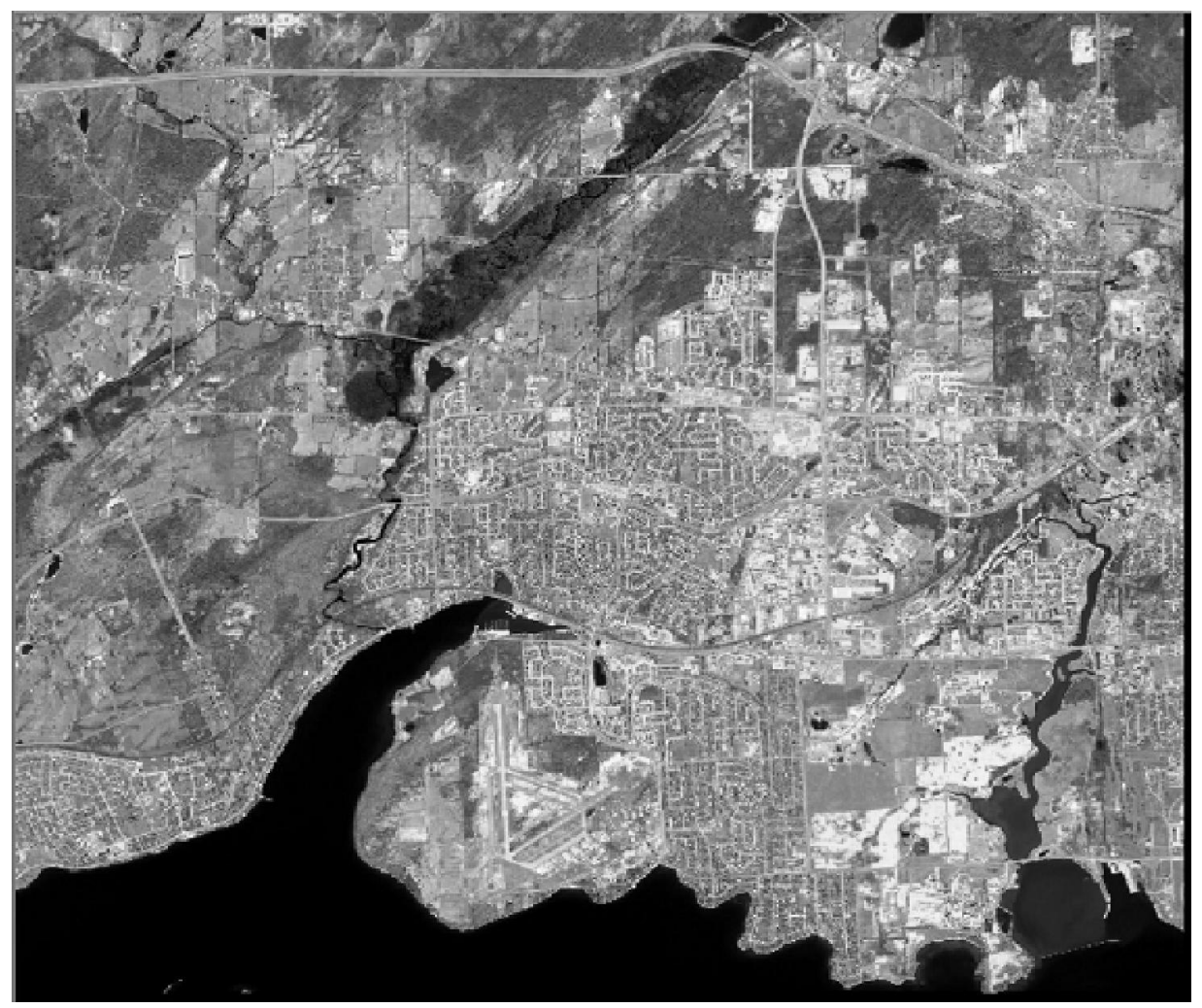

Figure 3. The near infrared (NIR) band of IKONOS image covering the study area.

The wavelet decomposition provides a set of coefficients at each pixel. For example, a one-level wavelet decomposition provides four coefficients, namely, the four spectral (or density) values in each of the four sub-images. An approach based on the direct use of wavelet decomposition coefficients is investigated in our study. The wavelet transform not only separates the trend from details as many other filtering methods do, but it also presents both sets of information at multiple scales. The wavelet coefficients in the approximation and detail sub-images are usually summarized by several indices that represent the underlying properties of the images. Widely used indices are statistical in nature, ranging from simple ones, such as mean and standard deviation, to more complex varieties, such as entropy measures (Pesaresi, 2000; MyInt, 2000).

\section{Methods}

\subsection{Study Area and Data Set}

An IKONOS multi-spectral image covering a portion of the western part of the city of Kingston, Ontario, Canada is used in this study. The IKONOS image was acquired on April 25,2000 with a spatial resolution of 4 meters and four spectral bands. The size of each spectral band is 2,363-by-2,517 pixels, covering an area of $9.45 \times 10.07 \mathrm{~km}^{2}$. The four spectral bands are the visible blue band (wavelength 0.45 to 0.53 $\mu \mathrm{m}$ ), visible green band (wavelength 0.52 to $0.61 \mu \mathrm{m}$ ), visible red band (wavelength 0.64 to $0.72 \mu \mathrm{m}$ ), and invisible near infrared band (wavelength 0.77 to $0.88 \mu \mathrm{m}$ ). Figure 3 shows the near infrared band of the IKNOS image.

\subsection{Classification Scheme}

Ten classes are defined in this study based on prior knowledge of the ground characteristics of the study area (Kingston western area), and based on a previous study of classification of this area (Chen, 2007). The ten classes are described in Table 1. Of these ten classes, some are land cover classes, such as coniferous forest, deciduous forest and water; the others are land use classes, such as residential and commercial.

\subsection{Feature Extraction}

Experiments described in this paper use three feature sets: a spectral-based feature set, a wavelet-based feature set, and a variance-based feature set. The spectral-based feature set is derived from full spectral bands of the study area. It uses a feature vector of length of four, with one feature from each band.

The variance band is generated from the spectral band through calculating the variance of an $n$-by- $n$ sliding window based on the image. A previous study by Chen (2007) showed that the NIR band has the most significant effect in the gene- 
Table 1. Land-Cover/Land-Use Classes in the Study Area

\begin{tabular}{|c|c|c|c|}
\hline Class No. & Class Name & $\begin{array}{l}\text { Normalized Homogeneity } \\
\text { Value }(0-1)\end{array}$ & Description \\
\hline 1 & Road /Transportation & 0.69 & Main street/transportation \\
\hline 2 & Residential & 0 & Mainly single houses in the study area \\
\hline 3 & Commercial & 0.45 & $\begin{array}{l}\text { Dominated by commercial building roof, parking lots, and industrial } \\
\text { complexes }\end{array}$ \\
\hline 4 & Coniferous forest & 0.68 & Vegetative communities dominated by evergreen coniferous trees \\
\hline 5 & Agriculture & 0.72 & Crops actively cultivated and irrigated \\
\hline 6 & Irrigated grassland & 0.89 & Big patches of land having grass only \\
\hline 7 & Wetland & 0.58 & Areas dominated by saturated soils and often standing water \\
\hline 8 & Cleaned/Vacant land & 0.64 & Vacant land or unused land \\
\hline 9 & Water body & 1 & $\begin{array}{l}\text { Mostly represented by Lake Ontario and creeks, as well as ponds } \\
\text { north of highway } 401\end{array}$ \\
\hline 10 & Deciduous forest & 0.66 & $\begin{array}{l}\text { Areas dominated by trees where the tree species shed foliage } \\
\text { simultaneously in response to seasonal change }\end{array}$ \\
\hline
\end{tabular}

* The calculation of Normalized Homogeneity Value is described in Section 3.4.

Table 2. Different Feature Combinations Used in This Study

\begin{tabular}{|c|c|c|c|c|}
\hline $\begin{array}{l}\text { Feature } \\
\text { set No. }\end{array}$ & Feature set & Description & $\begin{array}{l}\text { Abbr. of } \\
\text { feature set }\end{array}$ & $\begin{array}{l}\text { Length of } \\
\text { feature vector }\end{array}$ \\
\hline $\mathrm{A} 1$ & Spectral features & Use of the spectral-based feature set (B1-B4) only & $\mathrm{S}$ & 4 \\
\hline $\mathrm{A} 2$ & $\begin{array}{l}\text { One-level Wavelet } \\
\text { features }\end{array}$ & Use of one-level wavelet-based feature set (B1-B4) & $\mathrm{W}(\mathrm{I})$ & 16 \\
\hline A3 & $\begin{array}{l}\text { Two-level wavelet } \\
\text { features }\end{array}$ & Use of two-level wavelet-based feature set (B1-B4) & $\mathrm{W}(\mathrm{II})$ & 32 \\
\hline A4 & $\begin{array}{l}\text { Spectral + one-level } \\
\text { wavelet feature }\end{array}$ & $\begin{array}{l}\text { Use of combination of the spectral-based feature set (B1-B4) } \\
\text { and one-level wavelet-based feature set (B1-B4) }\end{array}$ & $\mathrm{SW}(\mathrm{I})$ & 20 \\
\hline A5 & $\begin{array}{l}\text { Spectral + two-level } \\
\text { wavelet features }\end{array}$ & $\begin{array}{l}\text { Use of the combination of the spectral-based feature set and } \\
\text { two-level wavelet-based feature set (B1-B4) }\end{array}$ & SW(II) & 36 \\
\hline A6 & Variance features & $\begin{array}{l}\text { Use of the variance-based feature set only (near infrared } \\
\text { band) }\end{array}$ & $\mathrm{V}$ & 1 \\
\hline A7 & $\begin{array}{l}\text { spectral + Variance } \\
\text { features }\end{array}$ & $\begin{array}{l}\text { Use of combination of spectral-based (B1-B4) and } \\
\text { variance-based feature set (single band) }\end{array}$ & SV & 5 \\
\hline A8 & $\begin{array}{l}\text { Variance }+ \text { one-level } \\
\text { wavelet features }\end{array}$ & $\begin{array}{l}\text { Use of the combination of the variance-based feature set (single } \\
\text { band) and one-level wavelet-based feature set (B1-B4) }\end{array}$ & VW(I) & 17 \\
\hline A9 & $\begin{array}{l}\text { Variance }+ \text { two-level } \\
\text { wavelet features }\end{array}$ & $\begin{array}{l}\text { Use of the combination of the variance-based feature set and the } \\
\text { two-level wavelet-based feature set (B1-B4) }\end{array}$ & VW(II) & 33 \\
\hline $\mathrm{A} 10$ & $\begin{array}{l}\text { Spectral }+ \text { one-level } \\
\text { wavelet }+ \text { Variance } \\
\text { features }\end{array}$ & $\begin{array}{l}\text { Use of the combination of the variance-based feature } \\
\text { set(B1-B4), one-level wavelet-based feature set (B1-B4), and } \\
\text { the spectral-based feature set (single band) }\end{array}$ & SW(I)V & 21 \\
\hline A11 & $\begin{array}{l}\text { Spectral + two-level } \\
\text { wavelet + Variance } \\
\text { features }\end{array}$ & $\begin{array}{l}\text { Use of the combination of the spectral-based feature set, } \\
\text { two-level wavelet-based feature set, and the variance-based } \\
\text { feature set (B1-B4) }\end{array}$ & SW(II) V & 37 \\
\hline
\end{tabular}

* B1-B4 represents the four spectral bands of the image

ration of the variance band. Therefore, the variance from the NIR band is used in this study. Several different window sizes including $3 \times 3,5 \times 5,7 \times 7,9 \times 9,11 \times 11$, and $13 \times 13$ are tested. The highest overall accuracy is achieved using a $7 \times 7$ window size. Thus, the variance band generated by the $7 \times 7$ window is used in the following comparison.

The wavelet-based feature set is derived from one or two level wavelet decomposition bands. The wavelet bands are derived from the spectral bands image. We create a one-level wavelet-based feature set, and a two-level wavelet-based feature set, respectively.

The feature sets used in this study are composed of individual feature sets or combinations of the three feature sets. Table 2 lists the different feature combinations and feature 
vector length tested in this study. In total, there are 11 classifications based on various feature sets.

\subsection{Training}

We use a minimum distance classifier (also called the nearest-neighbor classifier) (Duda et al., 2001). In the training stage, feature values are measured on training data, and these feature values are stored as a set of feature vectors for each class. To classify an unknown sample, the sample's feature vector is compared to all the stored feature vectors to find the closest one. The training samples are obtained by selecting 4by-4 image blocks for each class. Our choice of small block size is due to the limitation that it is very difficult to collect the Road class training sample with a size larger than 4-by-4, and we use the same training sample size for all classes. For wavelet-based features, the wavelet decomposition coefficient values of training samples are extracted using a one-level or two-level wavelet decomposition.

We define a homogeneity value, to describe the degree of similarity of pixel values within a class (or a region). The homogeneity value is measured as the inverse of the average variance value of training samples using the following formula:

$$
\text { HomogeneityValue }=\frac{1}{\operatorname{avg}\left(\sum_{i=1}^{M} \text { variance_training_sample }\right)}
$$

where $i$ represents the ith sampling block for a class; the variance_training_sample is the variance of the spectral values of the one training sample; $M$ represents the total number of sampling blocks for a class. The homogeneity value is calculated separately for each class. The homogeneity values are normalized and linearly scaled into the range of 0 to 1 , with the effect that the homogeneity values of Residential class and Water class are set to 0 and 1 , respectively. The normalized homogeneity values for each class are listed in Table 1 . The homogeneity value for the Commercial class is the average of the homogeneity values for its three sub-classes (commercial building roof, parking lots, and industrial complex).

\subsection{Classifier}

A minimum distance (MD) classifier is employed in all the classifications due to its simplicity. The ranges of values in the three feature sets are different. In order to meet the requirement of the MD classifier, all values in three feature sets are scaled (normalized) into the range 0 to 2047. A pixel is classified by measuring its feature values, and comparing them to those from training data (Duda et al., 2001). The Euclidean distances in feature space between a pixel and different classes are calculated, and then ordered. The pixel is assigned to its closest class.

\subsection{Accuracy Assessment}

The accuracy assessment is conducted by comparing the classified result of reference data with ground truth. In this study, the collection of the reference data includes sampling, visually interpreting the sampling points, and field-checking for reference data. First, 80 random points for each class were generated over the study area using a stratified random sampling method. In total, 800 points were generated. Visual interpretation and on-site field checking was conducted for each point. Due to changes in ground cover between dates of data acquisition and field-checking, some points can not be labelled with enough confidence. These points are removed. In the end, 706 points are kept.

An error matrix is generated for each classification. The overall accuracy and kappa coefficient, as well as user's and producer's accuracy of individual classes are derived from the error matrix.

\section{Result Analysis}

The overall accuracy and kappa coefficient for each classification set (A1 to A11) derived are listed in Table 3. Both overall accuracy and kappa coefficient show the same trend. The use of the wavelet-based feature set increases the overall accuracy for classes both with high homogeneity value and with low homogeneity value. Adding the variance-based feature set also dramatically increases the discrimination power for classes with low homogeneity.

Table 3. Overall Accuracy and Kappa Coefficient of Classifications with Different Feature Sets

\begin{tabular}{llll}
\hline No. & $\begin{array}{l}\text { Abbr. of Feature } \\
\text { sets }\end{array}$ & $\begin{array}{l}\text { Overall accuracy } \\
(\%)\end{array}$ & $\begin{array}{l}\text { Kappa } \\
\text { Coefficient }(\%)\end{array}$ \\
\hline A1 & S & 75.4 & 72.3 \\
A2 & W(I) & 79.7 & 77.1 \\
A3 & W(II) & 79.9 & 77.4 \\
A4 & SW(I) & 79.6 & 77.1 \\
A5 & SW(II) & 79.9 & 77.4 \\
A6 & V & 37.9 & 30.3 \\
A7 & SV & 79.1 & 76.4 \\
A8 & VW(I) & 85.5 & 82.9 \\
A9 & VW(II) & 86.7 & 85.1 \\
A10 & SW(I)V & 86.0 & 84.2 \\
A11 & SW(II)V & 89.1 & 87.7 \\
\hline
\end{tabular}

Tables 4 and 5 list the user's accuracy and producer's accuracy of classification with different feature sets (A.1 to A.11) for each individual class. In Tables 4 and 5, each column represents one of the 10 pre-defined classes, and each row represents classifications with different feature sets.

From the classification results, we see that most of the Road class is misclassified into Commercial class. The reason for this is that the Road class is composed of similar material as a commercial building roof or parking lot, such as concrete. The Road is linear in most cases, while the Commercial class is usually a polygonal region formed by a block of pixels. This is a very important feature to distinguish the Road class 
Table 4. User's Accuracy of Classification with Different Feature Sets

\begin{tabular}{lllllllllll}
\hline & Road & Residential & Commercial & Coniferous & Agriculture & Grassland & Wetland & Vacant & Water & Deciduous \\
A1 & 0.193 & 0.447 & 0.678 & 0.820 & 0.721 & 0.787 & 0.761 & 0.773 & 0.921 & 0.760 \\
A2 & 0.222 & 0.662 & 0.762 & 0.907 & 0.702 & 0.903 & 0.752 & 0.852 & 0.996 & 0.722 \\
A3 & 0.198 & 0.697 & 0.760 & 0.906 & 0.706 & 0.905 & 0.758 & 0.845 & 0.996 & 0.719 \\
A4 & 0.145 & 0.489 & 0.706 & 0.884 & 0.698 & 0.905 & 0.767 & 0.845 & 0.997 & 0.701 \\
A5 & 0.196 & 0.695 & 0.760 & 0.904 & 0.709 & 0.904 & 0.755 & 0.843 & 0.996 & 0.719 \\
A6 & 0.024 & 0.649 & 0.155 & 0.483 & 0.410 & 0.159 & 0.056 & 0.099 & 0.589 & 0.407 \\
A7 & 0.172 & 0.762 & 0.746 & 0.644 & 0.907 & 0.929 & 0.885 & 0.756 & 0.991 & 0.724 \\
A8 & 0.201 & 0.788 & 0.798 & 0.835 & 0.909 & 0.945 & 0.881 & 0.851 & 0.998 & 0.846 \\
A9 & 0.227 & 0.852 & 0.791 & 0.918 & 0.866 & 0.940 & 0.850 & 0.874 & 0.998 & 0.830 \\
A10 & 0.213 & 0.795 & 0.794 & 0.861 & 0.905 & 0.942 & 0.877 & 0.859 & 0.998 & 0.848 \\
A11 & 0.203 & 0.914 & 0.802 & 0.937 & 0.924 & 0.961 & 0.868 & 0.874 & 0.998 & 0.869 \\
\hline
\end{tabular}

* Feature sets A1 to A11 are defined in Table 2

Table 5. Producer's Accuracy of Classifications with Different Feature Sets

\begin{tabular}{lllllllllll}
\hline & Road & Residential & Commercial & Coniferous & Agriculture & Grassland & Wetland & Vacant & Water & Deciduous \\
A1 & 0.184 & 0.155 & 0.766 & 0.909 & 0.785 & 0.930 & 0.811 & 0.840 & 0.994 & 0.853 \\
A2 & 0.236 & 0.234 & 0.784 & 0.990 & 0.820 & 0.943 & 0.821 & 0.881 & 0.993 & 0.928 \\
A3 & 0.189 & 0.232 & 0.795 & 0.991 & 0.839 & 0.944 & 0.826 & 0.881 & 0.993 & 0.928 \\
A4 & 0.167 & 0.273 & 0.756 & 0.989 & 0.822 & 0.944 & 0.787 & 0.892 & 0.993 & 0.740 \\
A5 & 0.185 & 0.231 & 0.794 & 0.991 & 0.838 & 0.945 & 0.826 & 0.881 & 0.993 & 0.927 \\
A6 & 0.065 & 0.697 & 0.455 & 0.014 & 0.429 & 0.072 & 0.006 & 0.153 & 0.865 & 0.251 \\
A7 & 0.370 & 0.724 & 0.584 & 0.701 & 0.863 & 0.856 & 0.740 & 0.834 & 0.989 & 0.862 \\
A8 & 0.349 & 0.738 & 0.687 & 0.899 & 0.893 & 0.900 & 0.816 & 0.881 & 0.991 & 0.945 \\
A9 & 0.289 & 0.717 & 0.768 & 0.947 & 0.899 & 0.919 & 0.824 & 0.882 & 0.9937 & 0.936 \\
A10 & 0.349 & 0.738 & 0.705 & 0.909 & 0.896 & 0.906 & 0.820 & 0.880 & 0.992 & 0.947 \\
A11 & 0.367 & 0.880 & 0.721 & 0.995 & 0.903 & 0.915 & 0.809 & 0.882 & 0.992 & 0.937 \\
\hline
\end{tabular}

from the Commercial class. Algorithms specified for linear features can be used to separate the Road class and the Commercial class. However, this is not the focus of this paper, and we did not investigate this.

The spectral-based feature set has high discrimination power for classes with middle or higher homogeneity value, especially the Water class. For example, using only the spectral-based feature set, we achieve $92.1 \%$ user's accuracy and 99.4\% producer's accuracy for class Water; we also achieve $76.0 \%$ user's accuracy and $85.3 \%$ producer's accuracy for Deciduous forest. However, the spectral-based feature set is weak for detecting classes with low homogeneity value, such as the Residential class. Only $44.7 \%$ user's accuracy and $15.5 \%$ producer's accuracy are achieved for the Residential class.

The wavelet-based feature set improves the discrimination power for classes with low homogeneity value, compared with the spectral-based feature set. It also improves the over- all accuracy for classes with high homogeneity value. Compared with the spectral-based feature set, using the waveletbased feature set alone achieves 5\% accuracy improvement for classes with high homogeneity values and 3\% overall accuracy improvement for classes with low homogeneity values. The experimental results demonstrate that there is no significant difference between the use of the one-level waveletbased feature set and the two-level wavelet-based feature set.

The variance-based feature set alone has poor ability to discriminate most of the pre-defined classes except the Residential class, because it uses only the variance as the feature. Classes with middle or higher homogeneity value have similar variances. Therefore variance is not a good feature to distinguish classes with middle or higher homogeneity values.

However, adding the variance-based feature set to the wavelet-based feature set dramatically improves the classification accuracy for classes with low homogeneity value, especially the Residential class. The user's accuracy of Residential 
class rises from 44.7 to $91.4 \%$, and the producer's accuracy rises from 15.5 to $88.0 \%$ with the adding of the variance features

\section{Conclusions}

In this paper, we investigate the effectiveness of three individual feature sets and various combinations of the three feature sets for the classification of remotely-sensed images. A set of experiments are devised to provide a qualitative evaluation of classification results. Based on the experimental results, we draw the following conclusions:

1) The spectral-based feature set can achieve basic satisfactory results for classes with middle or higher homogeneity value. However, it has low discrimination power for classes with low homogeneity, especially the Residential class.

2) Compared with the spectral-based feature set, the wavelet-based ones (one-level or two-level) can slightly improve the classification accuracy. This is because wavelets have the capability to distinguish classes with different homogeneity value.

3) We did not find a significant difference in classification accuracy between one-level wavelets and two-level wavelets in this study.

4) The variance-based feature set alone has little discrimination power for all the classes. However, it plays a significant role in detecting classes with low homogeneity value when combined with other feature sets, such as the waveletbased feature set. Adding the variance-based feature set can dramatically improve the classification accuracy for classes with low homogeneity value.

Acknowledgments. This research is supported by discovery grants awarded to Chen and Blostein from Natural Sciences and Engineering Research Council of Canada, and by a grant from the Xerox Foundation.

\section{References}

Andrew, L. and Jian, F. (1993). Texture classification by wavelet packet signatures. IEEE Trans. Pattern Anal. Mach. Intell., 15(11), 1186-1191.

Arai, K. (1993). A classification method with a spatial-spectral variability. Int. J. Remote Sens., 14, 699-709.

Bian, L. (2003). Retrieving urban objects using a wavelet transform approach. Photogramm. Eng. Remote Sens., 69, 133-141.

Brodatz, P. (1966), Textures-A Photographic Album for Artists and Designers, Dover Publications, New York.

Chang, T. and Kuo, C.C.J. (1993). Texture analysis and classification with tree-structured wavelet transform. IEEE Trans. Image Process., 2(4), 429-441.

Chen, D. and Stow, D. (2003). Strategies for integrating information from multiple spatial resolutions into land use and land cover classification routines. Photogramm. Eng. Remote Sens., 69(11), 1279-1287.

Chen, D. (2007). A Standardized Probability Comparison Approach for Evaluating and Combining Pixel-based Classification Procedures. Photogramm. Eng. Remote Sens. (In press).

Conners, R.W. and Harlow, C. (1980). A theoretical comparison of texture algorithms. IEEE Trans. Pattern Anal. Mach. Intell., 2 (3), 204-222.

Duda, R., Hart, P. and Stork, D. (2001). Pattern Classification, John Wiley \& Sons, New York.

Edwards G., Landary, R. and Thomson, K.P.B. (1988). Texture analysis of forest regeneration sites in high-resolution SAR imagery, Proc. of the International Geosciences and Remote Sensing Symposium (IGARSS 88), ESA SP-284 (Paris, European Space Agency), pp. 1355-1360.

Fung, T. and Chan, K. (1994). Spatial composition of spectral classes: A structural approach for image analysis of heterogeneous landuse and land-cover types. Photogramm. Eng. Remote Sens., 60 (2), 173-180.

Gong, P. and Howarth, P.J. (1990). An assessment of some factors influencing multispectral land-cover classification. Photogramm. Eng. Remote Sens., 5(56), 507-603.

Gonzalez, R.C. and Woods, R.E. (2001). Digital Image Processing, Prentice Hall, NJ, pp. 349.

Jensen, J.R. (1996). Introductory Digital Image Processing-A Remote Sensing Perspective, Prentice Hall, NJ, pp. 316.

Lillesand, M. and Kiefer, W. (2000). Remote Sensing and Image Interpretation, John Wiley \& Sons, New York, pp. 532.

Mallat, S.G. (1989). A theory for multi-resolution signal decomposition: the wavelet representation. IEEE Trans. Pattern Anal. Mach. Intell., 11, 673-693.

Myint, S.W. (2000). Image texture analysis with high-resolution multispectral imagedata using wavelet transforms, 2000 University Consortium for Geographic Information Systems.

MyInt, S.W., Lam, N.S.N. and Tyler, J. (2002). An evaluation of four different wavelet decomposition procedures for spatial feature discrimination within and around urban areas. Trans. GIS, 6(4), 403-429.

MyInt, S.W., Lam, N.S.N. and Tyler, J. (2004). Wavelets for urban spatial feature discrimination: Comparisons with fractal, spatial autocorrelation, and spatial co-occurrence approaches. Photogramm. Eng. Remote Sens., 70(7), 803-812.

Pathak, V. and Dikshit, O. (2007). Factors affecting urban classifycation using textural features. Int. J. Remote Sens. (accepted).

Pesaresi, M. (2000). Texture analysis for urban pattern recognition using fine-resolution panchromatic satellite imagery. Geogr. Environ. Model., 4(1), 43-63.

Woodcock, C.E. and Harward, V.J. (1992). Nested-hierarchical scene models and image segmentation. Int. J. Remote Sens., 13, 31673187.

Young, R.K. (1993). Wavelet Theory and its Applications, Kluwer Academic Publishers, Boston.

Zhu, C. and Yang, X. (1998). Study of remote sensing image texture analysis and classification using wavelet. Int. J. Remote Sens., 19, 3197-3203. 